\title{
Multiple, independent colonizations of the Hawaiian Archipelago by the family Dolichopodidae (Diptera)
}

\author{
Kari Roesch Goodman $^{1}{ }^{\text {, Neal Evenhuis }}{ }^{2}$, Pavla Bartošová-Sojková ${ }^{3}$, Patrick Michael O'Grady ${ }^{\text {Corresp. }}{ }^{1}$ \\ 1 Department of Environmental Science, Policy and Management, University of California, Berkeley, Berkeley, California, United States \\ 2 Department of Natural Sciences, Bernice P. Bishop Museum, Honolulu, Hawaii, United States \\ 3 Institute of Parasitology, Biology Centre of the Czech Academy of Sciences, České Budějovice, Czech Republic \\ Corresponding Author: Patrick Michael O'Grady \\ Email address: ogrady@berkeley.edu
}

The family Dolichopodidae forms two of the four largest evolutionary radiations in the Hawaiian Islands across all flies: Campsicnemus (183 spp) and the Eurynogaster complex (66 spp). They also include a small radiation of Conchopus (6 spp). A handful of other dolichopodid species are native to the islands in singleton lineages or small radiations. This study provides a phylogenetic perspective on the colonization history of the dolichopodid fauna in the islands. We generated a multi gene data set including representatives from 11 of the 14 endemic Hawaiian dolichopodid genera to examine the history of colonization to the islands, and analyzed it using Bayesian and maximum likelihood phylogenetic methods. We used a subset of the data that included Conchopus and the eight genera comprising the Eurynogaster complex to estimate the first phylogenetic hypothesis for these endemic groups, then used Beast to estimate their age of arrival to the archipelago. The Eurynogaster complex, Campsicnemus and Conchopus are clearly the result of independent colonizations. The results strongly support the Eurynogaster complex as a monophyletic group, and also supports the monophyly of 4 of the 8 described genera within the complex (Adachia, Arciellia, Uropachys and Eurynogaster). Members of the family Dolichopodidae have been dispersing over vast distances to colonize the Hawaiian Archipelago for millions of years, leading to multiple independent evolutionary diversification events. The Eurynogaster complex arrived in the Hawaiian Archipelago 11.8 $\mathrm{Ma}$, well before the arrival of Campsicnemus (4.5 Ma), and the even more recent Conchopus (1.8 Ma). Data presented here demonstrate that the Hawaiian Dolichopodidae both disperse and diversify easily, a rare combination that lays the groundwork for field studies on the reproductive isolating mechanisms and ecological partitioning of this group. 


\section{Original Article}

Title:

Multiple, Independent Colonizations of the Hawaiian Archipelago by the Family Dolichopodidae (Diptera)

\section{Author names:}

1. Kari Roesch Goodman ${ }^{1, *}$

11 (First=Kari, Middle=Roesch, Last $=$ Goodman, as in K.R. Goodman)

2. Neal L. Evenhuis ${ }^{2}$

3. Pavla Bartošová-Sojková ${ }^{3}$

16

4. Patrick M. O’Grady ${ }^{1}$

${ }^{1}$ Department of Environmental Science, Policy and Management 130 Mulford Hall

University of California, Berkeley

Berkeley, CA 94720-3114

U.S.A.

${ }^{2}$ Bishop Museum

1525 Bernice Street

Honolulu, HI 96817-2704

U.S.A.

${ }^{3}$ Biology Centre of the Czech Academy of Sciences

Institute of Parasitology

Branišovská 31

České Budějovice, 37005

34 Czech Republic

*corresponding author: krgoodman@berkeley.edu, (510) 913-2109

37 Department of Environmental Science, Policy and Management

38130 Mulford Hall

39 University of California, Berkeley

40 Berkeley, CA 94720-3114

41

42 


\section{ABSTRACT}

45 The family Dolichopodidae forms two of the four largest evolutionary radiations in the Hawaiian

46 Islands across all flies: Campsicnemus (183 spp) and the Eurynogaster complex (66 spp). They

47 also include a small radiation of Conchopus (6 spp). A handful of other dolichopodid species are

48 native to the islands in singleton lineages or small radiations. This study provides a phylogenetic

49 perspective on the colonization history of the dolichopodid fauna in the islands. We generated a

50 multi gene data set including representatives from 11 of the 14 endemic Hawaiian dolichopodid

51 genera to examine the history of colonization to the islands, and analyzed it using Bayesian and

52 maximum likelihood phylogenetic methods. We used a subset of the data that included

53 Conchopus and the eight genera comprising the Eurynogaster complex to estimate the first

54 phylogenetic hypothesis for these endemic groups, then used BEAST to estimate their age of

55 arrival to the archipelago. The Eurynogaster complex, Campsicnemus and Conchopus are clearly

56 the result of independent colonizations. The results support the Eurynogaster complex as a

57 monophyletic group, and also supports the monophyly of 4 of the 8 described genera within the complex (Adachia, Arciellia, Uropachys and Eurynogaster). Members of the family

Dolichopodidae have been dispersing over vast distances to colonize the Hawaiian Archipelago for millions of years, leading to multiple independent evolutionary diversification events. The Eurynogaster complex is estimated to have arrived in the Hawaiian Archipelago 11.8 Ma, well before the arrival of Campsicnemus (4.5 Ma), and the even more recent Conchopus (1.8 Ma). easily, a rare combination that lays the groundwork for field studies on the reproductive isolating mechanisms and ecological partitioning of this group. 


\section{Keywords}

68 Colonization history, Diptera, Divergence dating, Dolichopodidae, Evolutionary radiation,

69 Hawaiian Islands, Long distance dispersal 


\section{INTRODUCTION}

Long distance dispersal from continental populations is critical to the formation of the

73 Hawaiian flora and fauna (Carson \& Kaneshiro, 1976; O’Grady et al., 2009), but is considered

rare. This infrequent arrival and establishment has led to a flora and fauna that is disharmonic

75

76

77

78 relative to those on the continents that served as sources (Gillespie \& Roderick, 2002). Recently, several studies (reviewed in Heaney, 2007; Bellemain \& Ricklefs, 2008) have shown that reverse colonization from Hawaii to continental landmasses is observed in birds (Filardi \& Moyle, 2005), plants (Harbaugh \& Baldwin, 2007) and insects (O'Grady \& DeSalle, 2008; Lapoint et al., 2014), suggesting that dispersal plays a larger role than previously thought and evidence is accumulating to indicate that movement to and from island systems is more common, especially at geological time scales (Heaney, 2007; Cibois et al., 2011; Hembry et al., 2013; Casquet et al., 2014). If a lineage is vagile enough to repeatedly colonize an area, there is a reduced chance that it will generate the reproductive isolation necessary to speciate and then radiate. Furthermore, if radiation does occur in a lineage and there is subsequent colonization of the area by close relatives, ecological theory would predict that the existing niches would be pre-empted (Hardin, 1960), rendering a second radiation unsuccessful. Thus, clear examples where a lineage colonizes and radiates repeatedly and substantially are rare.

The Hawaiian-Emperor Archipelago has a long and dynamic geological history, well isolated in the central Pacific Ocean far from any continental mass. It has been forming by the motion of the Pacific plate over a stationary hotspot (Wilson, 1963), generating an island chain that is at least 80 million years old (Clague \& Dalrymple, 1987; Duncan \& Keller, 2004; Sharp \& Clague, 2006). Island formation during this long history has been episodic, with some periods characterized by only few, low elevation atolls and reduced species diversity and other times with multiple high islands capable of supporting a diverse flora and fauna (Price \& Clague, 
95 1992). Many of the older islands that are now submerged or heavily eroded to small land masses

96 once provided the kind of high island habitat we are familiar with in the contemporary high

97 islands (Niihau, Kauai, Oahu, Molokai, Lanai, Maui, Kahoolawe and Hawaii), which have been

98 forming very recently - only over the past five million years (Clague \& Dalrymple, 1987;

99 Clague, 1996: Figure 1). The current high islands provide a rich array of habitats, ranging from

100 low to high elevation and very dry to very wet vegetation types.

101 All of the flora and fauna arrived to this dynamic archipelago via long distance dispersal

102 in an unlikely sequence of events in which taxa both managed to land on the islands and persist

103 once there (Zimmerman, 2001; Gillespie et al., 2012). Recent phylogenetic studies of Hawaiian

104 insects (Jordan et al. 2003; Mendelson \& Shaw, 2005; Shapiro et al. 2006; Medeiros et al. 2009;

105 Lapoint et al., 2011; Medeiros \& Gillespie, 2011; O’Grady et al., 2011; Haines \& Rubinoff,

106 2012; Bennett \& O'Grady, 2013; Bess et al., 2013; Goodman \& O'Grady, 2013; Lapoint et al.,

107 2013; Goodman et al., 2014; Haines et al., 2014; Lapoint et al., 2014), have begun to reveal the

108 history of colonization to and diversification within the Hawaiian Archipelago, and it appears

109 that history is somewhat idiosyncratic. Some large groups, such as Hawaiian Drosophilidae with

110 an estimated 1,000 species, colonized the Hawaiian Islands tens of millions of years ago. Other

111 diverse groups, such as Nesophrosyne leafhoppers, with 72 described and over 100 undescribed

112 species (Bennett \& O’Grady, 2011), and Campsicnemus flies with about 200 species (Goodman

113 et al., 2014) are young, dating to only a few million years. One thing is clear, however - very

114 few endemic Hawaiian plant or animal families have successfully colonized the islands multiple

115 times (e.g., Araliaceae; Plunkett et al. 1997; Costello \& Motley 2001) and in no case have any of

116 these generated two radiations of with more than 50 species each. 
117 Flies in the family Dolichopodidae are remarkable in that they have colonized the

118 Hawaiian Islands multiple times and still have managed to generate two of the largest

119 evolutionary radiations within the Hawaiian Diptera: Campsicnemus Haliday, 183 spp.

120 (Goodman et al., 2014), and the Eurynogaster complex, 66 spp. in eight genera (Evenhuis,

121 2005). In addition, they also generated a small radiation of 6 spp., Conchopus Takagi. In addition

122 to these three radiations, four other dolichopodid genera contain endemic species: Asyndetus (1),

123 Hydrophorus (2), Paraliancalus (2), and Thinophilus (1) (Table 1). Thus, the family

124 Dolichopodidae offers a unique opportunity to examine the timing and frequency of long

125 distance colonization events in the founding of the endemic Hawaiian fauna. While recent

126 molecular phylogenies of Dolichopodidae (e.g., Lim et al., 2010; Bernasconi et al., 2007) have

127 sampled some of these genera (e.g., Campsicnemus, Hydrophorus, Thinophilus), uneven

128 sampling between studies and the lack of Hawaiian exemplars makes it difficult to infer the

129 colonization history in detail. Furthermore, while the biogeography of Campsicnemus has been

130 studied (Goodman et al. 2014), the evolutionary relationships among the three radiations and the

131 monophyly and biogeography of the large Eurynogaster complex have never been examined.

132 The primary goal of this paper is to address the colonization history of the endemic

133 Hawaiian Dolichopodidae and assess how many colonization events have generated the present-

134 day diversity within this lineage. We sampled 11 of the 14 genera with endemic Hawaiian

135 species and included samples from across the family Dolichopodidae. We sequenced a

136 combination of five mitochondrial and two nuclear genes and used these data to estimate

137 colonization times using the Bayesian algorithm implemented in BEAST to infer the colonization

138 history of this family in Hawaii. With our sampling we also provide the first molecular 
139 phylogenetic analysis of the Eurynogaster complex, with which we assess the monophyly of this

140 lineages and its constituent genera.

141

142 MATERIALS \& METHODS Taxonomic sampling

144 Specimens were collected from 2004 to 2012 from sites across the Hawaiian Islands. The

145 bulk of Hawaiian Dolichopodidae species are endemic to high elevation $(900-1700 \mathrm{~m}$.) rain

146 forest habitats, and thus collecting efforts were concentrated in these areas. Other habitats (e.g.,

147 coastal strand, dry and mesic forests, alpine zone) were also sampled, including rocky beaches,

148 the only known habitat of Conchopus, Thinophilus, Asyndetus and Hydrophorus. We succeeded

149 in collecting specimens from 11 of the 14 Hawaiian dolichopodid genera with endemic species

150 known from the islands (Campsicnemus, Conchopus, Thinophilus and eight genera from the

151 Eurynogaster complex, Table S1a in Appendix S1 in Supporting Information). Data from the

152 Hawaiian Campsicnemus are included here from a previous study from our group, and are

153 described in Appendix A from Goodman et al. (2014). Material was collected by general

154 sweeping of vegetation and leaf litter, pan and Malaise trapping, and hand collecting. To

155 evaluate monophyly of and diversity within the Eurynogaster complex, we included

156 representatives from each of its eight constituent genera (Table 1; Evenhuis, 2005). No

157 Eurynogaster complex lineages were omitted from our sampling. All material was preserved in $15895 \%$ ethanol.

159 All material was identified using the most recent key to species in Tenorio (1969) and

160 Evenhuis (2005). Descriptions of new species from within the Eurynogaster complex discovered

161 as a result of this project are in preparation. Unpublished new species included in the study were 
162 given letters (e.g., Eurynogaster n. sp. A, B, C, etc.). In addition to the extracted specimens,

163 whenever possible, a series of conspecifics from the same site were also preserved in $95 \%$

164 ethanol. Voucher material has been deposited in the Bernice Pauahi Bishop Museum (Honolulu).

165 In addition, new sequences were generated for outgroup specimens from the non-endemic

166 Dolichopodidae: five specimens of Dolichopus exsul, two specimens of Chrysotus longipalpis,

167 and one specimen each of Condylostylus sp. and Tachytrechus angustipennis. Finally, sequences

168 from Hercostomus indonesianus were also downloaded from GenBank to include in the outgroup

169 (see Table S1a in Appendix S1). Access and collection permits were granted by the State of

170 Hawaii Department of Land and Natural Resources, the National Park Service (Hawaii

171 Volcanoes and Haleakala National Parks), Maui Land and Pineapple, East Maui Irrigation,

172 Parker Ranch, and The Nature Conservancy of Hawaii (Appendix S4).

173

Relationships within Dolichopodidae and colonization of the Hawaiian Islands

To address the question of whether the endemic dolichopodid fauna, including the three major radiations (Campsicnemus, the Eurynogaster complex and Conchopus) is the result of a single or multiple colonizations, new sequences were generated for the samples described above

179 (and in Table S1a in Appendix S1) and were combined with the entire data matrix generated

180 from the Goodman et al. (2014) Campsicnemus study. Genomic DNA was extracted from

181 individuals using a Qiagen DNeasy (Qiagen Inc.) DNA extraction kit, following the

182 manufacturer's protocol. Loci used are described in Table 2. Thermal cycling involved a simple

183 protocol for EF1a, a touchdown protocol for the mitochondrial genes and a nested reaction for

184 CAD (described in (Moulton and Weigmann, 2004)). The simple protocol began with an initial 
185 denaturing step at $95 \mathrm{C}$ for $4 \mathrm{~min}, 30$ cycles of $90 \mathrm{C}$ for $30 \mathrm{~s}, 54-58 \mathrm{C}$ for $30 \mathrm{~s}, 72 \mathrm{C}$ for $60 \mathrm{~s}$ and a

186 final extension for 5-10 $\min 72 \mathrm{C}$. The touchdown protocol began with an initial activation cycle

187 at $96 \mathrm{C}$ for $2.5 \mathrm{~min}$ followed by 25 cycles of $30 \mathrm{~s}$ denaturing at $96 \mathrm{C}, 30 \mathrm{~s}$ annealing through a

188 touchdown series starting from $55 \mathrm{C}$ and stepping down $0.4 \mathrm{C}$ per cycle, with $45 \mathrm{~s}$ extension at

189 72C. This was followed by 15 cycles of $30 \mathrm{~s}$ denaturing at 96C, $30 \mathrm{~s}$ annealing at $45 \mathrm{C}$ and $45 \mathrm{~s}$

190 extension at 72C. A final extension for $7 \mathrm{~min}$ at $72 \mathrm{C}$ ended the touchdown protocol. PCR

191 products were purified using Exo-SAP-IT (USB Corporation, Cleveland, OH) following standard

192 protocols, and the products were sent to the UC Berkeley DNA Sequencing Center for

193 sequencing in both directions on an ABI 3730 capillary sequencer. Eleven of the 14

194 dolichopodid genera with endemic species are represented. This yielded an alignment, referred to

195 as dataset A, containing 183 individuals and seven loci containing 4763 base pairs that was used

196 to assess deep temporal and biogeographic patterns within Hawaiian Dolichopodidae.

197 Phylogenetic Relationships within the Eurynogaster Complex

198 To assess the monophyly of the Eurynogaster complex and its component genera,

199 seventeen described, four new, and five possible new species (labeled as "sp. nr.") were included

200 in the phylogenetic analysis (Table 1). This matrix was designated as dataset B. Phylogenetic

201 analyses were performed on a data set consisting of 57 individuals (see Table S1 in Appendix

202 S1) and seven loci containing 5,908 base pairs. The more restricted taxon sampling in dataset B

203 was to maximize the completeness of the seven loci sampled, many of which weren't sampled in

204 the larger dataset A. Results between the two studies are largely congruent. Analyses were

205 conducted on each gene individually using maximum likelihood (ML, see below). Dataset B was

206 used to assess biogeographic patterns within the Eurynogaster complex of genera. 
criteria. For each of the ML and the BI analyses, the optimum partitioning schemes were calculated in PartitionFinder (Lanfear et al., 2012). The optimal partitioning scheme for the

210 combined analysis of Hawaiian Dolichopodidae (dataset A), was calculated from 18 original data

211 partitions $\left(16 \mathrm{~S}, 12 \mathrm{~S}\right.$ and $1^{\text {st }}, 2^{\text {nd }}$, and $3^{\text {rd }}$ codon positions for COI, COII, ND2, CAD, EF1 $\alpha$ and

212 one CAD intron region). Partitioning was calculated for the Eurynogaster complex dataset

213 (dataset B) from 20 original data partitions $\left(16 \mathrm{~S}, 12 \mathrm{~S}\right.$ and $1^{\text {st }}, 2^{\text {nd }}$, and $3^{\text {rd }}$ codon positions for

214 COI, COII, ND2, CAD, EF1 $\alpha \mathrm{A}$ and $\mathrm{EF} 1 \alpha \mathrm{B}$, intron regions for CAD, EF1 $\alpha \mathrm{A}, \mathrm{EF} 1 \alpha \mathrm{B}$ and ND2)

215 and selected using Bayesian Information Criterion (Table S2b in Appendix 2). For both datasets,

216 in the BI analyses, the best-fit model of sequence evolution for each data partition was also

217 selected using PartitionFinder (Table S2b in Appendix S2: Lanfear et al., 2012). Selection of

218 models and partitions proceeded as described above and these are reported in Table S1b in

219 Appendix S1. The ML analyses were performed on individual genes and on the concatenated 220 data sets in RAxML 3.7.2 (Stamatakis, 2006) on CIPRES (Miller et al., 2010) under the GTR

221 GAMMA model with 1,000 bootstrap replicates and a final search for the best tree. The BI

222 analyses were performed on the concatenated data sets using MrBayes 3.1.2 (Huelsenbeck \&

223 Ronquist, 2001) on CIPRES (Miller et al., 2010), with each analyses run for 30 million

224 generations with 2 independent runs each.

MCMC convergence diagnostics: For the BI analyses, stationarity was assessed within and convergence among each of the runs using several complimentary approaches: (1) convergence metrics provided by MrBayes 3.1.2 were checked (Huelsenbeck \& Ronquist, 2001) to ensure that the maximum standard deviation of split frequencies of any of the runs was under 0.05 and that the potential scale reduction factor for all parameters approached 1.0, and (2) the 
230 log-likelihood values for each run were plotted, the Effective Sample Sizes (ESS) were checked

231 to ensure there were an adequate number of independent samples, and the posterior distributions

232 of all parameters were examined using Tracer v.1.72 (Rambaut \& Drummond, 2012). Tracer

233 v.1.72 was also used to determine the burn-in phase by assessing each run's plot of log-

234 likelihood values over generations -stationarity was assumed to have been reached when the log

235 likelihood values reached a stable plateau. Finally, a 50\% majority rule consensus trees was

236 created from the resulting post burn-in trees.

237

238

239

240

241

242

243

244

245

246

247

248

249

250

251

\section{Divergence Time Estimation in the Eurynogaster complex:}

To estimate the age of the Eurynogaster complex lineage, divergence time estimation was performed on dataset B using a Bayesian relaxed-clock method implemented in BEAST 1.7.5 (Drummond et al., 2012) on CIPRES (www.phylo.org: Miller et al., 2010). The age of the Eurynogaster complex is unknown as representatives of the genus are not known outside of Hawaii and biota in the Hawaiian Islands does not fossilize well. There is a fossil available for one genus that has an endemic species in the Hawaiian Islands (Thinophilus Wahlberg: subfamily Hydrophorinae), but the wide range in ages of the fossils (Baltic amber Eocene/Oligocene; ca. 35-60 mya) compared with the very young ages of the islands make them unsuitable for use in this analysis. Instead, we used three biogeographic calibrations based on the island ages of Kauai, Maui and Hawaii (see Table S2a, Figure S2a in Supporting Information).

We then evaluated the impact of the Kauai calibration by running an analysis with only the Maui and Hawaii calibrations, and also ran two alternate analyses for comparison based on evolutionary rates, described in Appendix S2. 
253 Eurynogaster that exhibit a clear progression from older to younger islands (Oahu to Maui to

254 Hawaii). We also performed a maximum likelihood ancestral state reconstruction in MESQUITE

255 v.2.7.2 (Maddison \& Maddison, 2009) to assign ancestral areas to all nodes in the phylogeny.

256 We then selected a third well-supported node for calibration with a clear ancestral range

257 reconstruction to the oldest island of Kauai. All three nodes were calibrated with island dates

258 from Carson \& Clague (1995) (Table S2a, Figure S2a). While island calibrations have been

259 widely used for the estimation of divergence times in Hawaiian lineages (e.g., (Rubinoff \&

260 Schmitz, 2010; Lerner et al., 2011), it is plausible that divergence among populations occurred

261 prior to island emergence and was thus unrelated, or that it occurred well after the emergence of

262 the younger island (Heads, 2005). Standard deviations were chosen to accommodate some of this

263 uncertainty, including a biologically relevant timeframe during which habitat was likely

264 available on the islands, and the fact that the insects may have colonized the islands well before

265 or after they reached their peak heights (Table S2a).

266 Divergence time estimation was performed on dataset B described above. The same

267 seven gene concatenated data set (COI, COII, ND2, 12S, 16S, EF1 $\alpha$ and CAD) was analysed in

268 each of the analyses described here and in Appendix D. Partitions and the best fit models of

269 evolution for each partition were selected using BIC in PARTITIONFINDER (Lanfear et al., 2012).

270 Initial analyses indicated that these models overparameterized the data in that the ESS values

271 were extremely low for some parameters, despite being run with very long chains (beast-users

272 Google group discussions). For the final runs, all GTR models were changed to HKY (Table

273 C.2) and ESS increased significantly while divergence times and tree topology did not change.

274 Base frequencies were estimated from the data. The partitioning scheme in the divergence rate 
275 analyses differed only slightly from the island calibration analyses in that COI was assigned its

276 own partition (Table S1b). Site and clock models were unlinked and all partitions were analysed

277 using an uncorrelated lognormal relaxed clock except for the partition comprised of CAD

278 (positions $1 \& 2$ ) and the EF1 $\alpha$ intron, for which a strict clock could not be rejected and was thus

279 applied. The tree-shape prior was linked across partitions and the tree-shape prior was specified

280 as a Yule Process. The xml file was hand edited to include a starting tree, generated using

281 maximum likelihood in RAxML 3.7.2 (Stamatakis, 2006). Two independent MCMC searches

282 were conducted, each running for 50 million generations and sampled every 1000 generations.

283 The number of generations was selected to generate ESS values greater than 200 for each of the

284 parameters (Drummond et al., 2007). Convergence was assessed using TRACER v. 1.7.5 and trees

285 were summarized to one Maximum Clade Credibility (MCC) tree using Tree ANNOTATOR v.

$286 \quad$ 1.7.5 after removing a burn-in phase.

287

288

289

RESULTS \& DISCUSSION

\section{Phylogenetic relationships within the endemic Hawaiian Dolichopodidae}

The family Dolichopodidae includes more than 6,800 described species (Yang et al.,

291 2006) in 232 genera worldwide (Pape \& Thompson, 2013). A total of 29 genera are found in the

292 Hawaiian Islands. Of these, fifteen have been introduced in the past 150 years, most likely

293 through human activity, while the remaining fourteen genera present in the archipelago are

294 known to contain endemic Hawaiian taxa (Table 1). The relationships between Campsicnemus

295 and the Eurynogaster complex and the colonization history of these genera have remained an

296 open question, largely due to the difficulty of placing both in a subfamilial context. While

297 Campsicnemus is clearly placed in the subfamily Sympycninae, the placement of the 
298 Eurynogaster complex has been more difficult to ascertain (see Appendix S3 in Supporting

299 Information). Individual taxa have previously been described as members of the subfamilies

300 Sympycninae, Hydrophorinae, and Thinophilinae. Hardy \& Kohn (1964) considered

301 Eurynogaster and associated genera as part of the Sympycninae (see Figure S3a in Appendix 3).

302 Later, Evenhuis (2005) transferred the entire Eurynogaster complex to the Hydrophorinae. If the

303 current taxonomy placing these lineages in two separate subfamilies is correct, Campsicnemus

304 and the Eurynogaster complex represent independent colonizations to the Hawaiian Islands.

305 Molecular evidence demonstrates that the endemic Hawaiian dolichopodid fauna is

306 clearly the result of multiple colonizations to the archipelago (Figure 2, Figures S1a \& S1b in

307 Appendix S1). Several key nodes are well supported and allow us to infer the history of the

308 Hawaiian Dolichopodidae. Conchopus (posterior probability $(\mathrm{PP})=1$, bootstrap $(\mathrm{BS})=100$ : node

309 A, Figure 2), the Eurynogaster complex ( $\mathrm{PP}=1, \mathrm{BS}=100$ : node B, Figure 2), and Campsicnemus

$310(\mathrm{PP}=1, \mathrm{BS}=98$ : node $\mathrm{C}$, Figure 2$)$ are each supported as monophyletic with respect to other

311 dolichopodid genera. Another key node that is supported in both analyses ( $\mathrm{PP}=1$, $\mathrm{BS}=99$ : node

312 D, Figure 2) is the large clade that includes Campsicnemus and a number of non-Hawaiian

313 genera in the subfamily Sympycninae (e.g., Sympycnus, Teuchophorus) and does not include the

314 Eurynogaster complex. This demonstrates that there were at least three colonizations to Hawaii

315 by the family Dolichopodidae, one each by the three radiations: Campsicnemus, the

316 Eurynogaster complex, and Conchopus.

317 There is little support for the placement of Thinophilus, so its history of arrival to Hawaii

318 remains enigmatic (Figure 2, Figures S1a \& S1b in Appendix S1). This genus is known primarily

319 from the Indo-Pacific, with one species each known from the Galapagos Islands and the

320 Hawaiian Islands. Previously it has only been collected from rocky, wet sand on the south shores 
321 of Oahu (Carlton \& Eldredge, 2009). The specimen included in this study represents the first

322 record from Hawaii Island and suggests that directed collecting on the south shores of Maui and

323 Kauai may turn up additional populations.

324 Three genera that contain endemic taxa were not included in this study because they are

325 difficult to collect and we did not recover them in our sampling. While their placement must

326 await future work, their omission here does not change the result that the Hawaiian Islands have

327 been colonized multiple times. An additional issue is that support at many nodes in this

328 phylogeny is poor, owing partially to the large divergences between the subfamilies and the

329 incomplete taxon sampling with this enormous family. These issues are also seen in previously

330 published phylogenetic studies of dolichopodid relationships (Lim et al., 2010; Bernasconi et al.,

331 2007). The lack of support and long branches across most of the rest of this phylogeny preclude

332 identifying the specific sister lineages to the Hawaiian taxa (Figure 2).

\section{Phylogenetic Relationships in the Eurynogaster Complex}

335 The Eurynogaster complex, with 66 described species (Yang et al., 2006) and about a dozen

336 awaiting description, comprises the fourth most species-rich radiation of Hawaiian flies, after the

337 Drosophilidae, Campsicnemus, and Lispocephala (Muscidae). Although the Eurynogaster

338 complex is one of the largest radiations of Diptera in Hawaii, phylogenetic relationships in this

339 group have never been studied. This collection of genera are hypothesized to have been derived

340 from a single colonization to the Hawaiian Archipelago (Evenhuis, 2005). Little is known about

341 the biology of these species, but collecting observations suggest that species found on the forest

342 floor and on vegetation tend to be dull coloured, while species found in wet habitat, along seeps,

343 streams and on wet banks tend to have shiny metallic thoraces and/or abdomens. 
345 hypothesis of a monophyletic complex of related genera $(\mathrm{PP}=1, \mathrm{BS}=100$ : Figure 2$)$, as well as

346 support for several of the genera within this radiation. We focused on the smaller dataset (dataset

347 B) to address phylogenetic and biogeographic questions within the Eurynogaster genus complex.

348 Analyses of individual genes are presented in Figures S1e-S11 in Appendix S1, and final data

349 partitions and evolutionary models are reported in Table S1b in Appendix S1. Tree topologies

350 generated using ML and BI approaches of the concatenated dataset B were very similar; at well-

351 supported nodes, they are identical (Figures S1c \& S1d).

352 In Figure 1, the maximum clade credibility tree from the Bayesian analysis performed in

353 BEAST is used to display the patterns within the Eurynogaster complex, and the following PP

354 and BS supports are from the BI performed in MrBayes and ML analysis performed in RAxML

355 (shown in Figures S1c \& S1d). The Eurynogaster complex is split into two clades: Clade A

356 (Adachia + Elmoia + Sigmatineurum + Major, $\mathrm{PP}=1, \mathrm{BS}=100)$ and Clade B $($ Sweziella +

357 Arciellia + Uropachys + Eurynogaster, $\mathrm{PP}=1, \mathrm{BS}=100)$. Current sampling indicates that the

358 genus Adachia is monophyletic $(\mathrm{PP}=1, \mathrm{BS}=100)$ and sister to a well-supported clade $(\mathrm{PP}=1$,

359 BS=100) composed of the genera Elmoia, Sigmatineurum and Major (ESM Clade). Sampling

360 within the ESM clade is not extensive, with only a single representative each of Sigmatineurum

361 and Major. Two representatives of the genus Elmoia were sampled and our results indicate that

362 this genus is paraphyletic with respect to Sigmatineurum and Major. Denser sampling with the

363 ESM clade will be necessary to resolve the placement of the Elmoia taxa.

364 Clade B includes the large genus Eurynogaster, along with Arciellia, Uropachys and

365 Sweziella. Sweziella, represented by S. tergoprolixa from Maui, is the basal lineage within clade

366 B and sister to the lineage formed by Arciellia, Uropachys and Eurynogaster $(\mathrm{PP}=1, \mathrm{BS}=91$ : 
367 Figure 2). Current sampling indicates that the genus Arciellia and Uropachys are each

368 monophyletic $(\mathrm{PP}=1, \mathrm{BS}=100$ and $\mathrm{PP}=1, \mathrm{BS}=100$, respectively $)$ and sister to one another

$369(\mathrm{PP}=1, \mathrm{BS}=100)$. Eurynogaster is supported as monophyletic $(\mathrm{PP}=1, \mathrm{BS}=100)$. This genus is

370 confusing taxonomically and is in need of revision. There are three undescribed Eurynogaster

371 species that were discovered as part of this work, E. n. spp. A-C. There are also a number of taxa

372 that, while morphologically similar to named taxa, show significant sequence divergence from

373 the described species. This sometimes corresponds to samples having been taken from different

374 islands. For example, E. maculata from Oahu is quite different from the E. sp. nr. maculata

375 samples collected from Maui (E. sp. nr. maculata 141) and Hawaii Island (E. sp. nr. maculata

376115 and 126 ) - they are $3.9 \%$ and $3.6 \%$ divergent at COI, respectively. Furthermore, one

377 exemplar of $E$. maculata from Maui is quite similar to $E$. sp. nr. maculata 141 - it is identical at

378 COI - suggesting that cryptic species may exist within the concept of what we currently

379 recognize as E. maculata. This phenomenon is common in large evolutionary radiations in

380 Hawaii (e.g., Bennett \& O’Grady, 2011). Another species we sampled, E. cilifemorata, also

381 seems to be a complex of species sampled from Maui and Oahu. Additional sampling within

382 Eurynogaster, as well as thorough taxonomic revisions of the genera within this complex, will be

383 necessary to better delineate species within this rapidly evolving clade.

384 Finally, four new species within the Eurynogaster complex were discovered as a result of

385 this project, three within Eurynogaster and one within Sigmatineurum. An additional five

386 possible new species (Adachia - 1 species; Eurynogaster - 4 species) were identified (labeled as

387 "sp. nr.") and are in the process of examination to confirm their taxonomic status.

388

389 Arrival times and biogeography 

Clague, 2002). This ancient lineage arrived well before the formation of the current high islands about $5 \mathrm{Ma}$ and the arrival of Campsicnemus, which is estimated to have occurred approximately 4.6 Ma (Goodman et al., 2014). Early diversification into five of the eight contemporary genera took place in the older, now eroded, northwest Hawaiian Islands, and five colonizations of these ancestral lineages into the current main (high) islands are needed to explain the contemporary patterns of diversity. All of the diversification within the crown groups has occurred within the past 5 million years (Myr), the timeframe of the current high islands. The most speciose lineage within the Eurynogaster complex, the genus Eurynogaster, began diversifying approximately 2.6

402 (95\% HPD: 1.94-3.26) Ma, about the time Oahu and Maui Nui were forming. We estimate that 403 the small endemic dolichopodid genus Conchopus arrived quite recently - 1.77 (95\% HPD: 1.09-2.6) Ma (Figure 1). Within the Eurynogaster complex, a number of classic biogeographic patterns are evident, some of which are significantly different from what is observed in other large radiations.

407 First, a progression rule pattern (Hennig, 1966) is common in hotspot archipelagos where islands appear along a chronosequence. The typical progression rule pattern seen in Hawaii occurs when the most basally branching taxon is present on Kauai, the oldest island, with more recently

410 branching taxa present on the progressively younger islands of Oahu, Molokai, Maui and Hawaii

411 (Wagner \& Funk, 1995). While the progression rule is commonly observed in both the Hawaiian 412 Drosophila (Bonacum et al., 2005) and Campsicnemus (Goodman et al., 2014) lineages, it is less 
413 prevalent in Eurynogaster. Only a single lineage of the genus Eurynogaster shows a clear

414 progression from Oahu to Maui to Hawaii (Figure 1).

Another phenomenon observed in Hawaiian lineages is within-island diversification,

416 where species break up to diversify into new populations and eventually sibling species on the

417 same island. This has been thought to be an uncommon occurrence, in part because it is fairly

418 uncommon across the historically best-studied group in the islands, the Hawaiian Drosophila-

419 for whom diversification primarily occurs following inter-island dispersal. However, even within

420 this iconic group, there are examples and it has been very well studied in the sympatric sibling

421 pair D. silvestris and D. heteroneura (Carson, 1982; DeSalle et al., 1987; Price \& Boake, 1995).

422 Newer examples are now accumulating across taxonomic groups (e.g.: Goodman et al. 2012;

423 Eldon et al. 2013; Bennett \& O’Grady 2013; Liebherr, 2015), exposing how variable a process

424 diversification can be, and how dependent it is on the dispersal capabilities of the groups studied

425 (Price \& Wagner, 2004). The Eurynogaster complex shows at least five instances of within-

426 island diversification. Uropachys is a genus of 6 species only known from Kauai. Three

427 Uropachys species were sampled for this study and are supported as a monophyletic clade,

428 indicating they diversified there. This pattern is also observed in Adachia, where A. hispida and

429 A. apicenigra have both formed on Hawaii, and in several clades of the genus Eurynogaster

430 where diversification has occurred on Oahu and Hawaii. While it is possible that additional

431 sampling, and subsequent discovery of new species, may alter this inference, it is clear that

432 diversification within an island is a pattern seen in many other Hawaiian groups, including the

433 genus Campsicnemus (Goodman et al., 2014) and the well-studied Hawaiian Drosophila

434 (O’Grady et al. 2011).

435 
436 Colonization of and diversification within the Hawaiian Islands

437 It is clear that the endemic Dolichopodidae of Hawaii arrived to the archipelago in at

438 least three successful colonization to radiation sequences over the last 12 Myr (Eurynogaster

439 complex, 11.8 Ma; Campsicnemus, 4.6 Ma; Conchopus, 1.8 Ma)-demonstrating that dispersal to

440 and establishment within this remote island group is more common than has been documented in

441 other groups. This is fascinating because it means that three separate radiations occurred despite

442 the excellent dispersal capabilities of these animals. In order to multiply into radiations, they

443 must have been able to generate reproductive isolation rapidly enough to overcome gene flow

444 from their highly vagile conspecifics. Members of this family are known to have complicated

445 courtship behavior (Zimmer et al. 2003). Though this has never been studied in the Hawaiian

446 fauna, it may be a contributing factor to the development of reproductive isolation as has been

447 shown with the Hawaiian Drosophilidae (Kaneshiro, 1976; Price \& Boake, 1995), Laupala

448 (Grace \& Shaw, 2011) and Nesosydne (Goodman et al. 2015), and suggests fruitful research

449 directions. There seems to be no correlation between the age of colonization and the diversity of

450 each lineage.

451 MacArthur \& Wilson (1967) stated that "an island is closed to a particular species when

452 the species is excluded ... by competitors already in residence...”. The Hawaiian Islands were

453 clearly not closed to dolichopodid flies that arrived after the first wave $12 \mathrm{Ma}$. This suggests that,

454 at the arrival of each new lineage, there was still plenty of ecological opportunity available or

455 these insects are ecologically labile and able to adapt easily when faced with niches already

456 occupied by competitors. Both statements may be true.

457 Very little is known about the ecology of the Dolichopodidae in Hawaii, but they are

458 known to be predatory from observations elsewhere in the world (Ulrich, 2005). For the 
459 Eurynogaster complex (which only occur in Hawaii), there is only a single published account in

460 the literature that includes ecological observations (Williams, 1938). Despite the dearth of

461 ecological data available, we have some evidence to support the idea that the dolichopodids seem

462 to adapt easily. In our 2014 study, we used morphological colouring together with field

463 observations to infer that the Hawaiian Campsicnemus have rapidly diversified into three

464 ecological types: (1) brown, low vegetation and litter dwellers, (2) black water skaters and (3)

465 yellow canopy dwellers. Interestingly, the black water skaters and yellow canopy dwellers are

466 restricted to the Pacific. Furthermore, the yellow canopy dwellers are endemic to Hawaii

467 (Goodman et al. 2014). The Hawaiian Conchopus may have also undergone a shift in ecological

468 type. This lineage can be traced back to East Asia (Takagi, 1965), where they are known

469 primarily from barnacle colonies in the marine tidal zone, living in the interstices or in nearby

470 cracks in the rocks and feeding on tiny invertebrates (Sunose \& Sato, 1994). There are no native

471 barnacles in the Hawaiian Islands, and Conchopus there are known from puka (holes) in beach

472 rocks deriving from volcanic flows. Once established in this habitat, they radiated into six known

473 species.

474 Prevailing dogma among Hawaiian evolutionary biologists in the past 30 years has been

475 that colonization events to the archipelago are rare and colonization within the islands follow a

476 few well-defined patterns, such as the progression rule (Wagner \& Funk 1995). Recent

477 molecular phylogenetic studies are beginning to overturn these overly simplified notions

478 (Heaney 2007; Bellemain \& Rickliffs 2008), finding that colonization and diversification are

479 based on a combination of factors. These include characters linked to the dispersal and

480 adaptability of the lineage in question and the ecological and environmental context of the

481 islands when that lineage arrives. The current study highlighting the multiple colonizations that 
482 Dolicohpodidae have undergone in the past, and the specific patterns of diversification within the 483 Eurynogaster complex, further demonstrate that there are no simple "rules" and each 484 colonization event should be considered an independent event. The Hawaiian Dolichopodidae 485 are an intriguing example of repeated, overlapping evolutionary radiations, ripe for field studies 486 that can begin to untangle their propensity to speciate and ecological lability. 487 488

\section{ACKNOWLEDGEMENTS}

490

The following people are thanked for their contribution to this project: Bob Peck, Karl

491 Magnacca, Gordon Bennett, and Dan Polhemus for material; Sue Wang, Saya Wai and Crystal

492 Teng for help in the lab, and Dan Bickel and David Hembry for comments on the manuscript.

493 We also thank the State of Hawaii Department of Land and Natural Resources, the National Park

494 Service, Maui Land and Pineapple, East Maui Irrigation, Parker Ranch, and The Nature

495 Conservancy of Hawaii for access and permission to collect. This work was funded by NSF DEB

4960842348 to PMO. This paper constitutes Contribution No. 2016-xxx to the Hawaii Biological

497 Survey.

498

499 


\section{REFERENCES}

502 Bellemain, E. \& Ricklefs, R.E. (2008) Are islands the end of the colonization road?

503

504

505

506

507

508

509

510

511

512

513

514

515

516

517

518

519

520

521

522

523

524

525

526

527

528

529

530

531

532

533

534

535

536

537

538

539

540

541

542

543

544

545

Trends in Ecology and Evolution, 23, 461-468.
Bennett, G.M. \& O'Grady, P.M. (2011) Review of the native Hawaiian leafhopper genus Nesophrosyne (Hemiptera: Cicadellidae: Deltocephalinae) with descriptions of eight new species associated with Broussaisia arguta. Zootaxa 2805, 125.

Bennett, G.M. \& O'Grady, P.M. (2013) Historical biogeography and ecological opportunity in the adaptive radiation of native Hawaiian leafhoppers (Cicadellidae: Nesophrosyne). Journal of Biogeography, 40(8), 1512-1523.

Bernasconi, M.V., Pollet, M. \& Ward, P.I. (2007) Molecular systematics of Dolichopodidae (Diptera) inferred from COI and 12S rDNA gene sequences based on European exemplars. Invertebrate Systematics, 21, 453-470.

Bess, E.C., Catanach, T.A. \& Johnson, K.P. (2013) The importance of molecular dating analyses for inferring Hawaiian biogeographical history: a case study with bark lice (Psocidae: Ptycta). Journal of Biogeography, 41, 158-167.

Bonacum, J., DeSalle, R., O’Grady, P.M., Olivera, D.S.C.G., Wintermute, J. \& Zilversmit, M. (2001) New nuclear and mitochondrial primers for systematics and comparative genomics in Drosophilidae. Drosophila Information Service, 84, 201-204.

Bonacum, J., O’Grady, P.M., Kambysellis, M. \& DeSalle, R. (2005) Phylogeny and age of diversification of the planitibia species group of the Hawaiian Drosophila. Molecular Phylogenetics and Evolution. 37, 73-82.

Carlton, J.T. \& L.G. Eldredge (2009). Marine Bioinvasions of Hawai'i: The Introduced and Cryptogenic Marine and Estuarine Animals and Plants of the Hawaiian Archipelago. Bishop Museum Bulletin in Cultural and Environmental Studies 4, pp. 202. Bernice P. Bishop Museum, Honolulu.

Carson, H. L. 1982. Evolution of Drosophila on the newer Hawaiian volcanoes. Heredity, 48, 325.

Carson, H.L. \& Kaneshiro, K.Y. (1976) Drosophila of Hawaii - systematics and ecological genetics. Annual Review of Ecology and Systematics, 7, 311-345.

Carson, H.L. \& Clague, D.A. (1995) Geology and biogeography of the Hawaiian Islands. Hawaiian biogeography: evolution on a hotspot archipelago (ed. by W.L. Wagner and V.A. Funk), pp. 14-29. Smithsonian Institution Press, Washington, D.C.

Casquet, J., Bourgeois, Y.X., Cruaud, C., Gavory, F., Gillespie, R.G. \& Thebaud, C. (2015) Community assembly on remote islands: a comparison of Hawaiian and Mascarene spiders. Journal of Biogeography, 42, 39-50.

Cibois, A., Beadell, J.S., Graves, G.R., Pasquet, E., Slikas, B., Sonsthagen, S.A., Thibault, J., \& Fleischer, R.C. (2011) Charting the course of reed-warblers across the Pacific islands. Journal of Biogeography, 38, 1963-1975.

Clague, D.A. (1996) The growth and subsidence of the Hawaiian-Emporer volcanic chain. The origin and evolution of Pacific island biotas, New Guinea to eastern Polynesia: patterns and processes (ed. by A. Keast and S.E. Miller), pp. 35-50. SPB Academic Publishing Amsterdam.

Clague, D.A. \& Dalrymple, G.B. (1987) The Hawaiian-Emperor volcanic chain: part 1. Geologic evolution. United States Geological Survey Professional Paper, 1350, 5-54. 
546

547

548

549

550

551

552

553

554

555

556

557

558

559

560

561

562

563

564

565

566

567

568

569

570

571

572

573

574

575

576

577

578

579

580

581

582

583

584

585

586

587

588

589

590

Clary, D.O. \& Wolstenholme, D.R. (1985) The mitochondrial DNA molecule of Drosophila yakuba: Nucleotide sequence, gene organization and genetic code. Journal of Molecular Evolution, 22, 252-271.

Collins, K.P. \& Wiegmann, B.M. (2002) Phylogenetic relationships and placement of the Empidoidea (Diptera: Brachycera) based on 28S rDNA and EF-1alpha sequences. Insect Systematics and Evolution, 33, 421-444.

Costello, A. \& Motley, T. J. (2001) Molecular systematics of Tetraplasandra, Munroidendron and Reynoldsia sandwicensis (Araliaceae) and the evolution of superior ovaries in Tetraplasandra. Edinburgh Journal of Botany, 58, 229-242.

DeSalle, R. (1992) The origin and possible time of divergence of the Hawaiian Drosophilidae evidence from DNA-sequences. Molecular Biology and Evolution, 9, 905.

DeSalle, R., Freedman, R., Prager, E.M. \& Wilson, A.C. (1987) Tempo and mode of sequence evolution in mitochondrial DNA of Hawaiian Drosophila. Journal of Molecular Evolution, 26, 157-164.

Drummond, A.J., Ho, S.Y.W., Rawlence, N. \& Rambaut, A. (2007) A Rough Guide to BEAST 1.4 (Program Manual). In:

Drummond, A.J., Suchard, M.A., Xie, D. \& Rambaut, A. (2012) Bayesian phylogenetics with BEAUTi and the BEAST 1.7. Molecular Biology and Evolution, 29, 1969-1973.

Duncan, R.A. \& Keller, R.A. (2004) Radiometric ages for basement rocks from the Emperor Seamounts, ODP Leg 197. Geochemistry Geophysics Geosystems 5, Q08L03, doi:10.1029/2004GC000704.

Eldon, J., Price, J.P., Magnacca, K. \& Price, D.K. (2013). Patterns and processes in complex landscapes: testing alternative biogeographical hypotheses through intergrated analysis of phylogeography and community ecology in Hawaii. Molecular Ecology. 22, 3613-3628.

Evenhuis, N.L. (2005) A review of the genera comprising species of the genus Eurynogaster sensu Hardy \& Kohn, 1964 in Hawaii (Diptera: Dolichopodidae). Zootaxa, 1017, 39-60.

Filardi, C.E. \& Moyle, R.G. (2005) Single origin of a pan-Pacific bird group and upstream colonization of Australasia. Nature, 438, 216-219.

Gillespie R.G. \& Roderick G.K. (2002) Arthropods on islands: colonization, speciation, and conservation. Annual Review of Entomology 47, 595-632.

Gillespie, R.G., Baldwin, B.G., Waters, J.M., Fraser, C., Nikula, R., \& Roderick, G.K. (2012) Long-distance dispersal - a framework for hypothesis testing. Trends in Ecology \& Evolution, 27(1), 47-56.

Goodman, K.R., Welter, S.C. \& Roderick, G.K. (2012) Genetic divergence is decoupled from ecological diversification in the Hawaiian Nesosydne planthoppers. Evolution. 66, 27982813.

Goodman, K.R. \& O'Grady, P.M. (2013) Molecular phylogeny and biogeography of the Hawaiian craneflies Dicranomyia (Diptera: Limoniidae). PLoS One, 8(9):e73019.

Goodman, K.R., Evenhuis, N.L., Bartosoya-Sojkova, P. \& O’Grady, P.M. (2014) Diversification in Hawaiian long-legged flies (Diptera: Dolichopodidae: Campsicnemus): Biogeographic isolation and ecological adaptation. Molecular Phylogenetics and Evolution, 81, 232-241.

Goodman, K.R., Kelley, J.P., Welter, S.C., Roderick, G.K. \& Elias, D.O. (2015). Rapid diversification of sexual signals in Hawaiian Nesosydne planthoppers (Hemiptera: Delphacidae): the relative role of neutral and selective forces. Journal of Evolutionary Biology. 28(2), 415-427. 
591 Grace, J. L. and Shaw, K. L. (2011) Coevolution of male signal and female preference during

592

593

594

595

596

597

598

599

600

601

602

603

604

605

606

607

608

609

610

611

612

613

614

615

616

617

618

619

620

621

622

623

624

625

626

627

628

629

630

631

632

633

634

635

636 early lineage divergence of the Hawaiian cricket, Laupala cerasina. Evolution. 65, 21842196.

Haines, W.P. \& D. Rubinoff (2012) Molecular phylogenetics of the moth genus Omiodes Guenee (Crambidae: Spilomelinae), and the origins of the Hawaiian lineage. Molecular Phylogenetics and Evolution, 65, 305-316.

Haines, W.P., Schmitz, P., Rubinoff, D. (2014) Ancient diversification of Hyposmocoma moths in Hawaii. Naure Communications, 5, 3502, doi: 10.1038/ncomms4502.

Harbaugh, D. T. \& Baldwin, B. G. (2007) Phylogeny and biogeography of the sandalwoods (Santalum, Santalaceace): repeated dispersals throughout the Pacific. American Journal of Botany, 94, 1028-1040.

Hardin, J. (1960) The Competitive Exclusion Principle. Science. 131, 1292-1297.

Hardy, D.E. \& Kohn, M.A. (1964) Dolichopodidae. Insects of Hawaii. University of Hawaii Press, Honolulu, HI, vol 11, 1-256.

Heads, M. (2005) Dating nodes on molecular phylogenies: a critique of molecular biogeography. Cladistics, 21, 62-78.

Heany, L.R. (2007) Is a new paradigm emerging for oceanic island biogeography? Journal of Biogeography, 34, 753-757.

Hembry D.H., Kawakita A., Gurr N.E., Schmaedick M.A., Baldwin B.G., Gillespie R.G. (2013) Non-congruent colonizations and diversification in a coevolving pollination mutualism on oceanic islands. Proceedings of the Royal Society B, 280, 20130361.

Hennig, W. (1966) Phylogenetic Systematics. University of Illinois Press, Urbana, Illinois.

Huelsenbeck, J.P. \& Ronquist, F. (2001) MrBayes: Bayesian inference of phylogenetic trees. 17, 754-5.

Jordan, S., Simon, C. \& Polhemus, D. (2003) Molecular Systematics and Adaptive Radiation of Hawaii's Endemic Damselfly Genus Megalagrion (Odonata: Coenagrionidae). Systematic Biology. 52(1), 89-109.

Kaneshiro, K.Y. (1976) Ethological Isolation and Phylogeny in the Planitibia Subgroup of Hawaiian Drosophila. Evolution. 30(4), 740-745.

Lanfear, R., Calcott, B., Ho, S.Y.W. \& Guindon, S. (2012) PartitionFinder: combined selection of partitioning schemes and substitution models for phylogenetic analyses. Molecular Biology and Evolution, 29, 1695-1701.

Lapoint, R.T., Gidaya, A., \& O'Grady, P.M. (2011) Phylogenetic relationships in the spoon tarsus subgroup of Hawaiian Drosophila; Conflict and concordance between gene trees. Molecular Phylogenetics and Evolution, 58, 492-501.

Lapoint, R.T., O'Grady, P.M. \& Whiteman, N.K., (2013) Diversification and Dispersal of the Hawaiian Drosophilidae: the evolution of Scaptomyza. Molecular Phylogenetics and Evolution, 69(1), 95-108.

Lapoint, R.T., Magnacca, K.N., and O'Grady, P.M. (2014) Phylogenetics of the antopocerusmodified tarsus clade of Hawaiian Drosophila: Diversification across the Hawaiian Islands. PLoS ONE, 9(11), e113227.

Liebherr, J.K. (2015) The Mecyclothorax beetles (Coleoptera, Carabidae, Moriomorphini) of Haleakala, Maui: Keystone of a hyperdiverse Hawaiian radiation. Zookeys. 544, 1-407.

Lim, G.S., Hwang, W.S., Kutty, S.N., Meier, R. \& Grootaert, P. (2010) Mitochondrial and nuclear markers support the monophyly of Dolichopodidae and suggest a rapid origin of the subfamilies (Diptera: Empidoidea). Systematic Entomology, 35, 59-70. 
637 Lerner, H.R.L., Meyer, M., James, H.F., Hofreiter, M. \& Fleischer, R.C. (2011) Multilocus

638 Resolution of Phylogeny and Timescale in the Extant Adaptive Radiation of Hawaiian

639 Honeycreepers. Current Biology, 21, 1-7.

640

641

642

643

644

645

646

647

648

649

650

651

652

653

654

655

656

657

658

659

660

661

662

663

664

665

666

667

668

669

670

671

672

673

674

675

676

677

678

679

680

MacArthur, R.H. \& Wilson, E.O. (1967). The Theory of Island Biogeography. pp. 203. Princeton University Press, Princeton.

Maddison, W.P. \& Maddison, D.R. (2009) Mesquite: a modular system for evolutionary analysis. Version 2.72. http://mesquiteproject.org.

Medeiros, M.J., Davis, D., Howarth, F.G. \& Gillespie, R.G. (2009) Evolution of cave living in Hawaiian Schrankia (Lepidoptera: Noctuidae) with description of a remarkable new cave species. Zoological Journal of the Linnean Society. 156, 114-139.

Medeiros, M.J. \& Gillespie, R.G. (2011) Biogeography and the evolution of flightlessness in a radiation of Hawaiian moths (Xyloryctidae: Thyrocopa). Journal of Biogeography. 38, 101-111.

Mendelson, T.C. \& Shaw, K.L. (2005). Sexual Behavior: rapid speciation in an arthropod. Nature, 433, 375-376.

Miller, M.A., Pfeiffer, W. \& Schwartz, T. (2010) "Creating the CIPRES Science Gateway for inference of large phylogenetic trees" Proceedings of the Gateway Computing Environments Workshop (GCE), 14 Nov. 2010 (ed by, pp. 1-8. New Orleans, LA.

Moulton, J.K. \& Weigmann, B.M. (2004) Evolution and phylogenetic utility of cad (rudimentary) amond Mesozoic-aged Eremoneuran Diptera (Insecta). Molecular Phylogenetics and Evolution, 31, 363-378.

Nishida, G.N. (2002) Hawaiian terrestrial arthropod checklist. Fourth edition. Bishop Museum Technical Report 22, 1-313.

O'Grady, P.M. and DeSalle, R. (2008) Out of Hawaii: The biogeographic history of the genus Scaptomyza (Diptera: Drosophilidae). Biology Letters 4(2), 195-199.

O’Grady, P.M., Magnacca, K.N. \& Lapoint, R.T. (2009) Drosophila. Encyclopedia of Islands (ed. by R.G. Gillespie and D.A. Clague), pp. 232-235. University of California Press, Berkeley, CA.

O’Grady, P.M., Lapoint, R.T., Bonacum, J., Lasola, J., Owen, E., Wu, Y., \& DeSalle, R. (2011) Phylogenetic and ecological relationships of the Hawaiian Drosophila inferred by mitochondrial DNA analysis. Molecular Phylogenetics and Evolution. 58, 244-256.

Pape, T. \& Thompson, F.C. (editors). (2013) Systema Dipterorum. Version 1.5. Available at: http://www.diptera.org/. [Last accessed 16 October 2014].

Plunkett, G. M., Soltis, D. E. \& Soltis, P. S. (1997) Clarification of the relationship between Apiaceae and Araliaceae based on matK and rbcL sequence data. American Journal of Botany. 84, 565-580.

Price, D. K. \& Boake, C. R. B. (1995). Behavioral reproductive isolation in Drosophila silvestris, D. heteroneura and their F1 hybrids (Diptera: Drosophilidae). Journal of Insect Behaviour 8, 595-616.

Price, J.P. \& Clague, D.A. (2002) How old is the Hawaiian biota? Geology and phylogeny suggest recent divergence. Proceedings of the Royal Society of London, B, 269, 24292435.

Price, J.P. \& Wagner, W.L. (2004) Speciation in Hawaiian angiosperm lineages: cause, consequence, and mode. Evolution. 58, 2185-2200. 
681

682

683

684

685

686

687

688

689

690

691

692

693

694

695

696

697

698

699

700

701

702

703

704

705

706

707

708

709

710

711

712

713

714

715

716

717

718

719

Rambaut, A. \& Drummond, A.J. (2012) Tracer v1.7.2, obtained from the "Workshop on Molecular Evolution", August 2011. Tracer v1.5 is available from http://beastbioedacuk/Tracer.

Rubinoff, D. \& Schmitz, P. (2010) Multiple aquatic invations by an endemic, terrestrial hawaiian moth radiation. Proceedings of the National Academy of Sciences, 107, 5903-5906.

Shapiro, L.H., Strazanac, J.S. \& Roderick, G.K. (2006) Molecular phylogeny of Banza (Orthoptera: Tettigoniidae), the endemic katydids of the Hawaiian Archipelago. Molecular Phylogenetics and Evolution. 41, 53-63.

Sharp, W.D. \& Clague, D.A. (2006) 50-Ma Initiation of Hawaiian-Emperor Bend Records Major Change in Pacific Plate Motion. Science, 313, 1281-1284.

Simon, C., Frati, F., Beckenbach, A., Crespi, B., Liu, H. \& Flook, P. (1994) Evolution, weighting, and phylogenetic utility of mitochondrial gene sequences and a compilation of conserved polymerase chain reaction primers. Annals of the Entomological Society of America, 86, 651-701.

Stamatakis, A. (2006) RAxML-VI-HPC: Maximum Likelihood-based Phylogenetic Analyses with Thousands of Taxa and Mixed Models. Bioinformatics, 22, 2688-2690.

Sunose, T. \& Sato, M. (1994) Morphological and Ecological Studies on a Marine Shore Dolichopodid Fly, Conchopus borealis Takagi (Diptera, Dolichopodidae). Japanese Journal of Entomology. 62(4), 651-660.

Takagi, S. (1965) A contribution to the knowledge of the marine shore dolichopodidae of Japan (Diptera). Insecta Matsumaurana. 27(2), 49-84.

Tenorio, J.M., 1969. Supplement, Diptera: Dolichopodidae, Appendix (Phoridae), Volume 11. Insects of Hawaii, Honolulu, $\mathrm{v}+73 \mathrm{pp}$.

Ulrich, H. (2005) Predation by adult Dolichopodidae (Diptera): a review of literature with an annotated prey-predator list. Studia Dipterologica, 11, 369-403.

Williams, F.X. (1938) Biological studies in Hawaiian water-loving insects part III, Diptera or flies B. Asteiidae, Syrphidae and Dolichopodidae. Proceedings of the Hawaiian Entomological Society, X, 281-315.

Wagner, W.L. \& Funk, V.A. (editors) (1995) Hawaiian biogeography: evolution on a hot spot archipelago. Smithsonian Institution Press, Washington and London, 467.

Wilson, J.T. (1963) A Possible Origin of the Hawaiian Islands. Canadian Journal of Physics, 41, 863-870.

Yang, D., Zhu, Y., Wang, M. \& Shang, L. (2006) World Catalog of Dolichopodidae (Insecta: Diptera). China Agricultural University Press, Beijing.

Zimmer, M., Diestelhorst, O. \& Lunau, K. (2003) Courtship in long-legged flies (Diptera: Dolichopodidae): function and evolution of signals. Behavioral Ecology. 14, 526-530.

Zimmerman, E.C. (2001) Volume 1 Reissue - Introduction with a new preface and dedication. Insects of Hawaii, Honolulu, 206 pp. 


\section{FIGURES}

722

723 Figure Legends

724

725 Figure 1. Maximum clade credibility tree summarizing BEAST analysis of the Eurynogaster 726 complex with geologic history of the archipelago. Node bars are the $95 \%$ highest posterior

727 density intervals of the divergence time estimate - bars that are offset are not to scale. Islands

728 that each specimen was collected from are shown next to each tip.

729 Figure 2. Majority rule consensus tree summarizing Bayesian analysis of the endemic

730 Dolichopodidae, with the large radiations, Eurynogaster complex and Campsicnemus collapsed.

731 Bayesian posterior probabilities (Mr. Bayes) and bootstrap supports from the maximum

732 likelihood analysis (RAxML) are displayed as ovals. 


\section{Table $\mathbf{1}$ (on next page)}

Table 1

Composition and status of Dolichopodidae fauna of Hawaii. Genera with endemic species are boldface. 
1 Table 1. Composition and status of Dolichopodidae fauna of Hawaii. Genera with endemic

2 species are boldface.

\begin{tabular}{|c|c|c|c|c|}
\hline Genus & $\begin{array}{l}\text { Total spp. in } \\
\text { Hawaii }\end{array}$ & $\begin{array}{l}\text { Number of } \\
\text { endemic spp. }\end{array}$ & $\begin{array}{l}\text { Number of non- } \\
\text { endemic spp. }\end{array}$ & $\begin{array}{c}\text { Number of described spp, } \\
\text { included in this study } \\
\text { (undescribed spp.) } \\
\text { [included from outside } \\
\text { Hawaii] }\end{array}$ \\
\hline Achradocera & 2 & 0 & 2 & 0 \\
\hline Amblypsilopus & 1 & 0 & 1 & 0 \\
\hline Asyndetus & 1 & 1 & 0 & 0 \\
\hline Austrosciapus & 1 & 0 & 1 & 0 \\
\hline Campsicnemus & 183 & 183 & 0 & $70[14]$ \\
\hline Chrysosoma & 2 & 0 & 2 & 0 \\
\hline Chrysotus & 1 & 0 & 1 & $1(1)[1]$ \\
\hline Conchopus & 6 & 6 & 0 & 3 \\
\hline Condylostylus & 1 & 0 & 1 & 1 \\
\hline Dactylomyia & 1 & 0 & 1 & 0 \\
\hline Diaphorus & 1 & 0 & 1 & 0 \\
\hline Dolichopus & 1 & 0 & 1 & 1 \\
\hline \multicolumn{5}{|l|}{$\begin{array}{l}\text { Eurynogaster } \\
\text { complex }\end{array}$} \\
\hline Adachia & 6 & 6 & 0 & $2(1)$ \\
\hline Arciellia & 3 & 3 & 0 & 2 \\
\hline Elmoia & 8 & 8 & 0 & 2 \\
\hline Eurynogaster & 23 & 23 & 0 & $6(7)$ \\
\hline Major & 1 & 1 & 0 & 1 \\
\hline Sigmatineurum & 11 & 11 & 0 & 1 \\
\hline Sweziella & 7 & 7 & 0 & 1 \\
\hline Uropachys & 7 & 7 & 0 & 3 \\
\hline Hydrophorus & 2 & 2 & 0 & 0 \\
\hline Krakatauia & 1 & 0 & 1 & 0 \\
\hline Medetera & 1 & 0 & 1 & 0 \\
\hline Paraliancalus & 2 & 2 & 0 & 0 \\
\hline Pelastoneurus & 1 & 0 & 1 & 0 \\
\hline Sympycnus & 1 & 0 & 1 & $1[5]$ \\
\hline Syntormon & 1 & 0 & 1 & $1[5]$ \\
\hline Tachytrechus & 1 & 0 & 1 & 1 \\
\hline Thinophilus & 1 & 1 & 0 & 1 \\
\hline
\end{tabular}




\section{Table 2 (on next page)}

Table 2

Primer names and references. Mitochondrial primer numbers correspond to the location in the Drosophila yakuba mitochondrial genome (Clary \& Wolstenholme, 1985). Sequences with no reference were designed as a part of this study. 
1 Table 2. Primer names and references. Mitochondrial primer numbers correspond to the location 2 in the Drosophila yakuba mitochondrial genome (Clary \& Wolstenholme, 1985). Sequences with 3 no reference were designed as a part of this study.

\begin{tabular}{|c|c|c|c|}
\hline Primer name & Length & Genome & Reference or Sequence \\
\hline $\begin{array}{l}\text { Cytochrome Oxidase I (COI): } \\
2183 \text { or } 2640 \text { and } 3041\end{array}$ & 829 & mitochondrial & (Bonacum et al., 2001) \\
\hline $\begin{array}{l}\text { Cytochrome Oxidase II (COII): } \\
3037 \text { and } 3771\end{array}$ & 681 & mitochondrial & (Bonacum et al., 2001) \\
\hline $\begin{array}{l}\text { NADH Dehydrogenase } 2 \text { (ND2): } \\
192 \text { and } 732\end{array}$ & 527 & mitochondrial & (Bonacum et al., 2001) \\
\hline $16 \mathrm{~S}$ & 530 & mitochondrial & (DeSalle, 1992) \\
\hline $12 \mathrm{~S}$ & 559 & mitochondrial & $\begin{array}{l}\text { F14233, R14922 (Simon et al., 1994) } \\
\text { 12S_exF: 5'-TCC AGT ACA TCT ACT ATG TTA CG-3' } \\
\text { 12S_inF: 5'-ATG TGT RCA TAT TTT AGA GC-3' } \\
\text { 12S inR: 5'-TAT TRG CTA AAT TTG TGC CAG C-3' }\end{array}$ \\
\hline $\begin{array}{l}\text { rudimentary }(\mathrm{CAD}) \\
\text { nested reaction: } \\
320 \mathrm{~F} \text { and } 843 \mathrm{R} \text {, } \\
338 \mathrm{~F} \text { and } 680 \mathrm{R}\end{array}$ & 896 & nuclear & (Moulton \& Weigmann, 2004) \\
\hline $\mathrm{EF} 1 \alpha \mathrm{A}$ & 1036 & nuclear & $\begin{array}{l}\text { EF4 and EF5 (Collins \& Wiegmann, 2002) } \\
\text { EFF: 5'-CNC CTG GCC ATC GTG ATT TC-3' } \\
\text { EFR: 5'-CAG CAT CTC CYG ATT TGA TGG C-3' }\end{array}$ \\
\hline $\mathrm{EF} 1 \alpha \mathrm{B}$ & 858 & nuclear & $\begin{array}{l}\text { EFF_B: 5'-GAT TAC TGG TAC ATC TCA AGC-3' } \\
\text { EFR_B: 5'-TAG CAG CAT CYC CYG ATT-3' }\end{array}$ \\
\hline
\end{tabular}

4

5 
Figure 1

Figure 1

Maximum clade credibility tree summarizing BEAST analysis of the Eurynogaster complex with geologic history of the archipelago. Node bars are the $95 \%$ highest posterior density intervals of the divergence time estimate - bars that are offset are not to scale. Islands that each specimen was collected from are shown next to each tip.

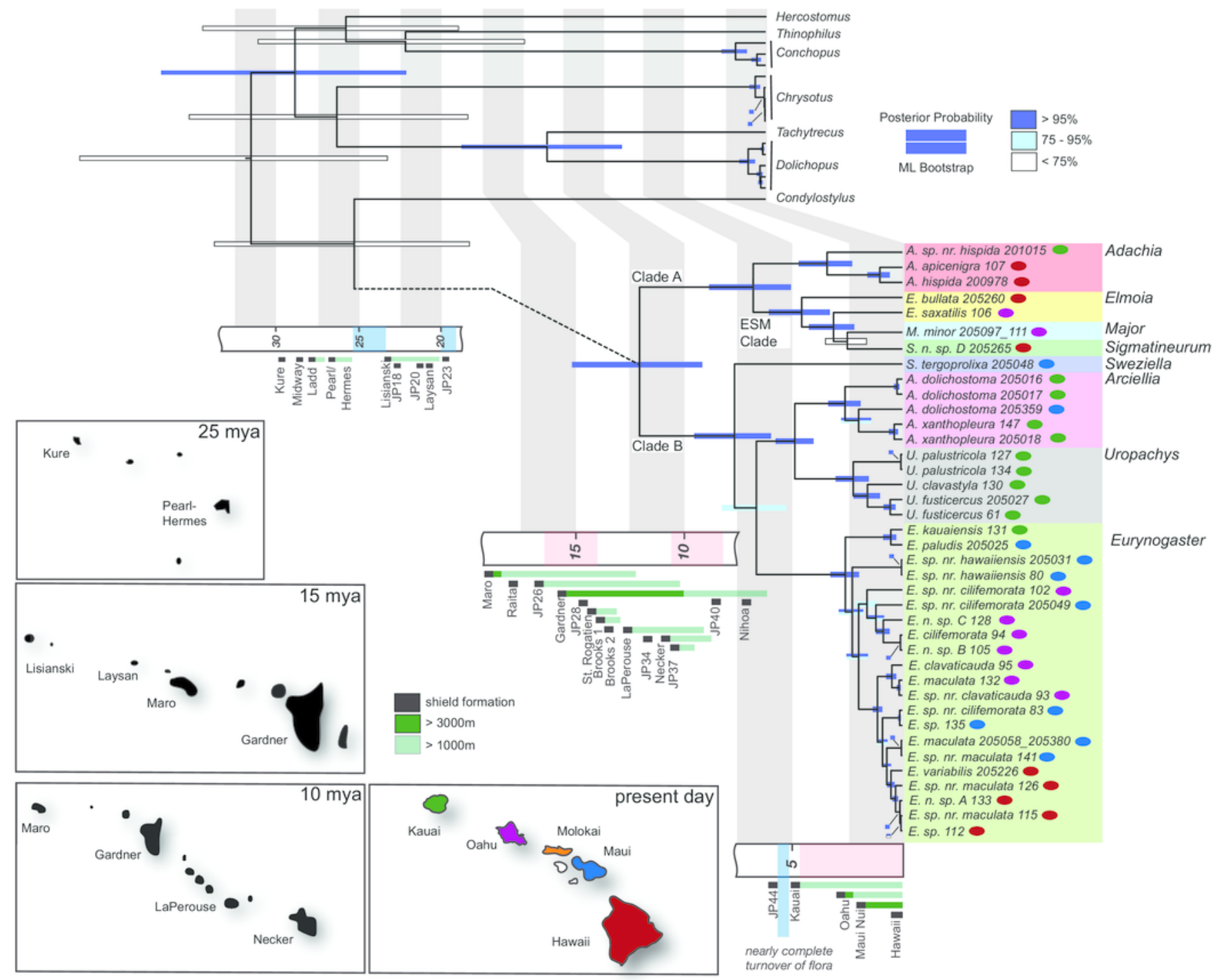




\section{Figure 2}

Figure 2

Majority rule consensus tree summarizing Bayesian analysis of the endemic Dolichopodidae, with the large radiations, Eurynogaster complex and Campsicnemus collapsed. Bayesian posterior probabilities (Mr. Bayes) and bootstrap supports from the maximum likelihood analysis (RAxML) are displayed as ovals.

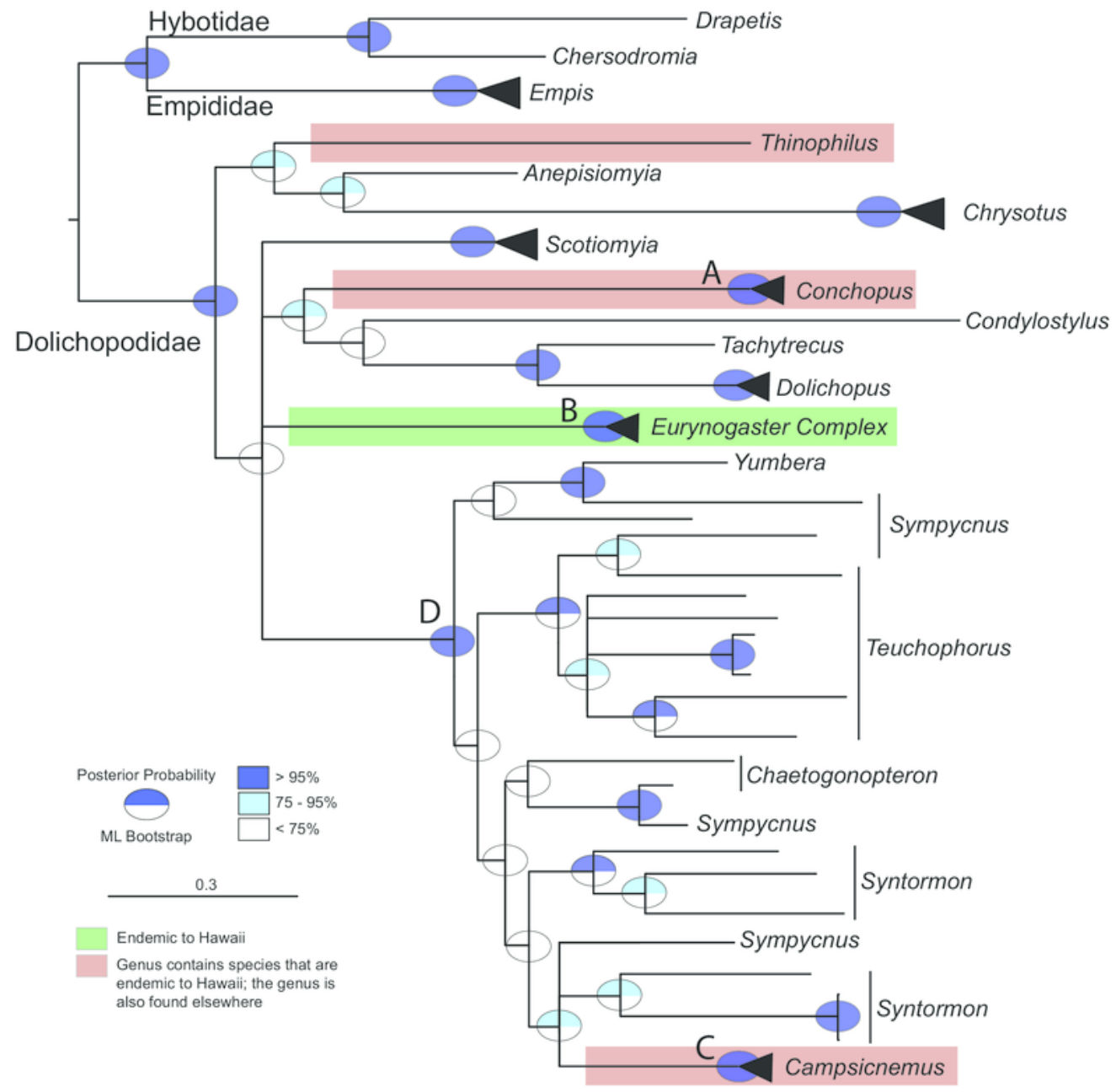

